This item was submitted to Loughborough's Research Repository by the author.

Items in Figshare are protected by copyright, with all rights reserved, unless otherwise indicated.

\title{
Multiplexed, label-free detection of biomarkers using aptamers and Tunable Resistive Pulse Sensing (AptaTRPS).
}

PLEASE CITE THE PUBLISHED VERSION

http://dx.doi.org/10.1016/j.bios.2015.02.011

PUBLISHER

(C) Elsevier B.V

VERSION

AM (Accepted Manuscript)

\section{PUBLISHER STATEMENT}

This work is made available according to the conditions of the Creative Commons Attribution-NonCommercialNoDerivatives 4.0 International (CC BY-NC-ND 4.0) licence. Full details of this licence are available at: https://creativecommons.org/licenses/by-nc-nd/4.0/

\section{LICENCE}

CC BY-NC-ND 4.0

\section{REPOSITORY RECORD}

Billinge, Emily R., and Mark Platt. 2019. "Multiplexed, Label-free Detection of Biomarkers Using Aptamers and Tunable Resistive Pulse Sensing (aptatrps).”. figshare. https://hdl.handle.net/2134/16838. 
Multiplexed, label-free detection of biomarkers using aptamers and tunable resistive pulse sensing (AptaTRPS)

Emily R. Billinge and Mark Platt*

Centre of Analytical Sciences, Department of Chemistry, School of Science.

Loughborough University, Loughborough, Leicestershire, LE11 3TU, United

Kingdom

*Correspondence should be sent to m.platt@lboro.ac.uk 


\section{Abstract:}

Diagnostics that are capable of detecting multiple biomarkers are improving the accuracy and efficiency of bioassays. In previous work we have demonstrated the potential of an aptamer-based sensor (aptasensor) utilising Tunable Resistive Pulse Sensing (TRPS). Here, we have advanced the technique identifying key experimental designs for potential POC assays. The assay utilised superparamagnetic beads, and using TRPS monitored their translocations through a pore. If the surfaces of the beads are modified with an aptamer, the frequency of beads (translocations/minute) through the pore can be related to the concentration of specific proteins in the solution. Herein, we have demonstrated the successful use of TRPS to observe the binding of two proteins, to their specific aptamers simultaneously. We describe a series of experiments illustrating key factors which we believe are integral to bead-based assays and demonstrate a general method for a multiplexed assay. In summary, we have explored the effects of beads size, concentration, potential bias, $\mathrm{pH}$ and aptamer affinity to enhance the sensitivity and practically of a TRPS aptasensor. The method utilises the fact the binding of the aptamer to the protein results in a change in charge density on the bead surface, the isoelectric point of the protein then dominates the mobility of the beads, creating a multiplexed assay termed AptaTRPS. By alteration of the applied potential to the instrument it is possible to produce a positive signal in a simple multiplexed assay.

Keywords: Aptasensor, aptamers, biomarkers, Tunable Resistive Pulse Sensing, Point of Care 


\section{Introduction:}

In recent decades there has been an increase in interest in rapid, affordable and user-friendly diagnostic techniques; driven by the rise in interest in personalised medicine, reduced assay times and the desire to provide home diagnostics(Chan et al., 2013). To make personalised medicine available to everyone, it is necessary to minimise the cost and labour intensity of assays so that they are affordable and available regardless of geographical location(Yager et al., 2008). Additionally, it is also desirable to minimise the time taken to yield results of an assay and to move towards point-of-care (POC) diagnostics and monitoring to improve decision-making. Key considerations in the choice of an appropriate technology are the ability to multiplex, assay time, cost, reliability and simplicity(Song et al., 2014).

Gold standard methods of analysis still heavily rely on PCR or antibody based technologies. A number of concerns remain with antibodies, as they: add cost and complexity to any assay; require the use of animals or cell culture for their generation; are susceptible to batch-to-batch variations; cause difficulties in handling and storage; and can have less than ideal sensitivity(Ledur et al., 1995; Tombelli et al., 2005). Their integration in POC technologies often gives the device a limited shelf life, and test substrates require refrigeration. A viable and accessible alternative to antibodies, addressing these concerns, would be a transformative technology. Aptamers are rapidly gaining interest as one alternative to antibodies(Fang and Tan, 2010; Levy-Nissenbaum et al., 2008; Mayer, 2009; Rowe et al., 2009). They offer an increased stability and ease of synthesis, they are far more cost-effective and reliable than their antibody counterparts and can easily be incorporated into diagnostic platforms. Full reviews on aptasensors can be found(Chiu and Huang, 2009; Cho et al., 2009; de-los-Santos-Álvarez et al., 2008; Famulok and Mayer, 2011) and whilst they have been criticised for repetition of choice in targets(Cho et al., 2009), this is likely due to authors keeping with tried and tested high affinity ligands to enable greater comparative power between new platforms.

One of the more recent technologies to implement aptamers is Resistive Pulse Sensing (RPS)(Rotem et al., 2012). A more recent form of RPS uses a polyurethane elastomeric membrane and is known as Tunable Resistive Pulse Sensing (TRPS)(Platt et al., 2012) in which the pore is able to be mechanically manipulated in 
real time to alter pore geometry(Roberts et al., 2010; Vogel et al., 2011). In brief, the set up and theory for RPS technologies is as follows: a stable ionic current is established by two electrodes, separated by a pore; as beads/analytes translocate the pore they temporarily occlude ions, leading to a transient decrease in potential known as a "blockade event", examples of which can be seen in Figure 1C. In the current arrangement, the pore is mounted laterally so that particles typically move from the upper fluid cell into the lower fluid cell, aided by an inherent pressure head due to $40 \mu \mathrm{l}$ of liquid in the upper fluid cell of approximately 50Pa (Willmott et al., 2012), and a positive or negative bias is applied via an electrode under the pore (see figure 1B). By monitoring changes in full width half maximum (FWHM), peak magnitude $\left(\Delta \mathrm{i}_{\mathrm{p}}\right)$ and peak frequency (events/min) it is possible to elucidate the zeta potential(Vogel et al., 2012), size(Vogel et al., 2011), and concentration(Willmott et al., 2010) of colloidal dispersions in situ, as described in more detail elsewhere(Vogel et al., 2011). TRPS is well suited to detect DNA and DNA-protein interactions; previous work has provided proof-of-concept studies on the technology(Billinge et al., 2014) in which the velocity and frequency of aptamertagged beads changes upon the addition of the target analyte as the aptamer folds to a specific conformation whereby the target binds, shielding the surface charge and altering double layer structure, as has been demonstrated with the target thrombin(Billinge et al., 2014). A significant development to this method would be the ability to detect multiple proteins at once.

In the present study we develop an aptasensor to haemostatic proteins Vascular Endothelial Growth Factor (VEGF) and Platelet Derived Growth Factor (PDGF) which are both involved in various disease processes such as cancer(Wei et al., 2003), atherosclerosis(Matsumoto and Mugishima, 2006), and Alzheimer's(Tarkowski et al., 2002). Although circulating levels of VEGF have been found to be variable between individuals, between 9 - $150 \mathrm{ng} / \mathrm{L}(J e l k m a n n, 2001)$, VEGF levels have been demonstrated to be elevated in several aggressive types of cancer, such as ovarian(Li et al., 2004), haematoloical(Belgore et al., 2001) and gastric carcinoma(Karayiannakis et al., 2002) and VEGF measurement has been highlighted as both a potential prognostic or diagnostic marker(Jelkmann, 2001) and for comparison between pre- and post-operative cancer states(Karayiannakis et al., 2002; Li et al., 2004). PDGF levels have also been found to be elevated in several 
different forms of cancer and has recently been added to the Glasgow Prognostic Index, which measures inflammatory mediators, as an important indication of tumour and significant predictor of survival(Hamilton et al., 2014). Previous studies have measured both VEGF and PDGF levels in cancer patients undergoing anti-VEGF treatment(Madsen et al., 2012), however this was performed over two different systems, increasing the cost and time considerations of the assay.

To monitor two analytes simultaneously in one-step analysis we use the ability of TRPS to provide accurate particle-by-particle analysis. Its ability to accurately calculate the beads size as it translocates the pore, allows the data from each particle set to be separated according to their size. By using two differently sized beads and separately functionalising them with anti-VEGF or anti-PDGF aptamers, as displayed in figure $1 \mathrm{~A}$, it is possible to separate the individual signals based on $\Delta \mathrm{i}_{\mathrm{p}}$, figure $1 \mathrm{C}$, and monitor changes to their frequency and speed, relating them back to the target analyte concentration. 


\section{A}

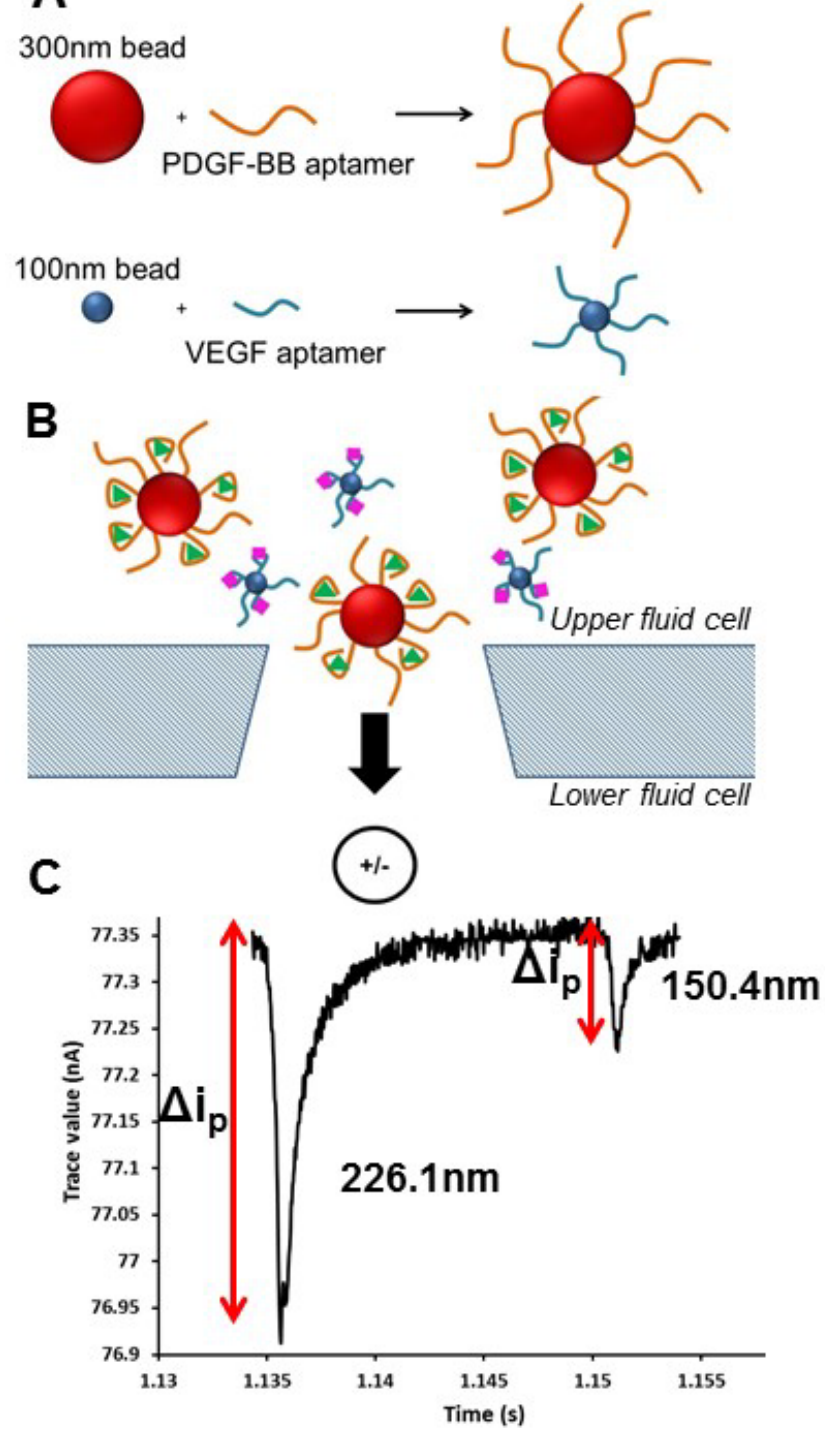

Figure 1: A Schematic of the multiplexed experimental process; two beads of different sizes are conjugated to either VEGF or PDGF. B Beads are incubated with a combination of concentrations of VEGF and PDGF for 30 minutes; as they bind to the target their velocity and frequency through the pore is monitored. C Recorded "resistive peaks" for two different sized beads; the blockade magnitude $\left(\Delta i_{p}\right)$ of each peak is proportional to the volume of each bead and can therefore be calibrated to provide the diameter of each individual bead. 


\section{Materials and Methods:}

\section{Chemicals and reagents}

The following chemicals were sourced from Sigma Aldrich, UK, without any further purification unless otherwise stated: Phosphate Buffered Saline (PBS - P4417), Tween 20 (P1379), Bovine Serum Albumin (BSA - A2153), and thrombin (T7513). DNA sequences were obtained from Sigma Aldrich's custom oligonucleotides service as lyophilised powders and made up to a stock concentration of $100 \mathrm{pmol} / \mu \mathrm{L}$ : 5' CAG GCT ACG GCA CGT AGA GCA TCA CCA TGA TCC TG-Biotin-3' (antiPDGF-BB aptamer)(Deng et al., 2013), 5' ATA CCA GTC TAT TCA ATT GGG CCC GTC CGT ATG GTG GGT GTG CTG GCC AG-Biotin-3' (VEa5 anti-VEGF aptamer)(Hasegawa et al., 2008). 5' TGT GGG GGT GGA CGG GCC GGG TAG ATT TTT-Biotin-3' (V7t1 anti-VEGF aptamer)(Nonaka et al., 2010). Human recombinant PDGF-BB and human VEGF 165 were purchased from Life Technologies, UK (PHG0044 and PHC9394, respectively). Water purified to a resistivity of $18.2 \mathrm{M} \Omega$ cm (Maxima) was used to make all solutions unless otherwise specified and 1XPBST (0.05\% Tween) was used as the buffer.

\section{Beads}

Streptavidin modified superparamagnetic beads of $120 \mathrm{~nm}$ (bio-adembeads streptavidin plus 0321) and $300 \mathrm{~nm}$ (bio-adembeads streptavidin 0313) in diameter were purchased from Ademtech (France). Binding capacities of $120 \mathrm{~nm}$ and $300 \mathrm{~nm}$ beads according to the manufacturer were $3512 \mathrm{pmol} / \mathrm{mg}$ and $477 \mathrm{pmol} / \mathrm{mg}$ respectively. Carboxyl beads of known concentration and diameter (SKP200; mode diameter 200nm) were sourced from Izon Sciences (Oxford, UK) to be used as a calibrant.

\section{Aptamer functionalization}

Aptamers were received as a lyophilised powder and made up to a stock concentration of $100 \mu \mathrm{M}$ with deionised water, these were then separated into aliquots for storage in the freezer so that sequences were only frozen and thawed once. The 120 and $300 \mathrm{~nm}$ beads were vortexed for 1 minute, sonicated for 1 minute and diluted to a concentration of $3 \times 10^{9}$ beads $/ \mathrm{mL}$ in PBST. The streptavidin coated beads were incubated with a 10-fold excess of the desired biotinylated aptamer at room temperature on a rotary wheel for 30 minutes to allow the streptavidin to react with the biotinylated ends of the DNA. The functionalised beads were then removed from the rotary wheel and centrifuged for 2 minutes at $10000 \mathrm{rpm}$ before being 
placed onto a MagRack (Life Sciences). After 2 minutes the beads had formed a visible cluster in the sample vial adjacent to the magnet and the solution was carefully removed and replaced with an equal volume of PBST - this wash stage was performed twice to remove any excess aptamer which would lower the sensitivity of the binding assay. These could then be stored in the fridge.

\section{Individual protein measurements}

Beads functionalised with the aptamers were divided into several vials, to which varying volumes of the protein stock solution was added to each vial to create samples of aptamer modified beads mixed with a range of protein concentrations. In all cases the volume of the solution was adjusted and kept constant by adding PBST. Each sample was left on a rotary wheel for 30 minutes to allow the protein to associate before being introduced into the TRPS instrument.

\section{Multiplexed analysis of PDGF-BB and VEGF}

$120 \mathrm{~nm}$ beads with V7t1 aptamer and $300 \mathrm{~nm}$ beads with PDGF-BB aptamer were prepared as described above at a bead concentration of $6 \times 10^{9}$ beads/mL. $50 \mu \mathrm{L}$ of each of the functionalised beads were placed into vials and combinations of concentrations of PDGF-BB and VEGF 165 were added. Each sample was left on a rotary wheel for 30 minutes to allow the protein to associate before being introduced into the TRPS instrument. Each sample was analysed via TRPS for the maximum run time of 10 minutes. The peak traces were calibrated and separated according to their size for independent analyses. Each individual blockade trace was examined for shape and directionality before being included in the sample set.

\section{Tunable Resistive Pulse Sensing (TRPS)}

Measurements were made with the Izon qNano system, purchased from Izon Sciences (Oxford, UK) which incorporates the fluid cell, stretching apparatus, data recording and analysis software (Control Suite V2.2.2.117). The pores used were designated "np200" by the manufacturer and are described as most suitable for detecting beads in the range $100-300 \mathrm{~nm}$. A stretch of $46 \mathrm{~mm}$ and voltage of $+/-0.4$ $\checkmark$ were chosen so that blockade events were clearly visible above the level of noise $(<10 p A)$; these conditions were maintained throughout the experiments so that data was comparable. $80 \mu \mathrm{L}$ of electrolyte buffer (PBS) was carefully pipetted into the lower fluid cell, taking care not to introduce bubbles. The upper fluid cell was then attached to the instrument and $40 \mu \mathrm{L}$ of PBST was added to enable confirmation of a stable baseline and clean pore. When a positive current is applied $(+0.4 \mathrm{~V})$ peaks 
are visible as a downward deflection from the baseline, when a negative current is applied (-0.4 V) peak were visible as upward deflections of the baseline.

\section{Data Analysis}

\section{Removal of reverse peaks}

Raw data was analysed by examining each individual blockade trace visually using the Izon ControlSuite V2.2.2.117 Software. When resistance was highest at the beginning of this peak and tailed toward the baseline with time this was considered a "normal" translocation. When the current increased gradually and then dropped sharply this was considered a "reverse" translocation and removed from subsequent data processing. Examples of these are shown in Supplementary Figure 1.

\section{Extraction of the particle rate for each bead population}

Each bead records a blockade magnitude that reflects its volume, for example 120 $\mathrm{nm}$ Ademtech beads typically produce a blockade magnitude of $0.17 \mathrm{nA}$ with the pore setup used here. Data which had been visually inspected as described above was then calibrated with beads of known diameter and narrow distribution. Prior to any analysis the Ademtech beads were calibrated separately to accurately measure the range of particle sizes in the provided batches and these ranges were used to provide filter information for data processing of experimental samples. Peaks recorded as being between 100 and $200 \mathrm{~nm}$ were recorded as "120 nm" and peaks between 210 and $400 \mathrm{~nm}$ were recorded as "300 nm". Data was then extracted as a .csv file containing data for each particle and the particle rate was then calculated by counting the number of beads observed divided by the recording time. 


\section{Results and Discussion:}

A

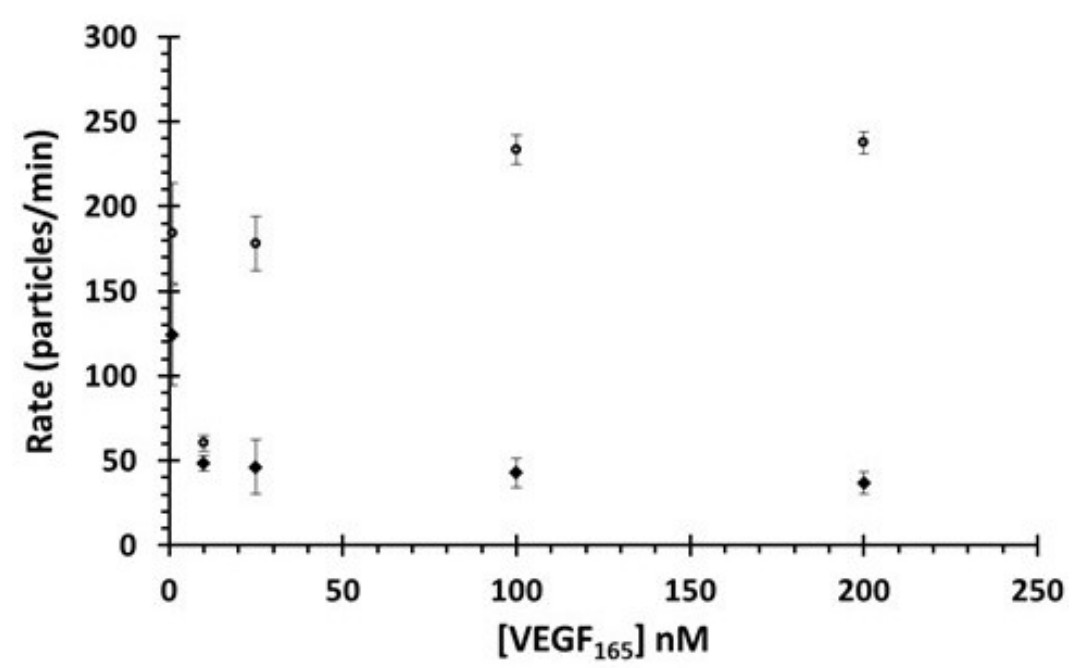

B

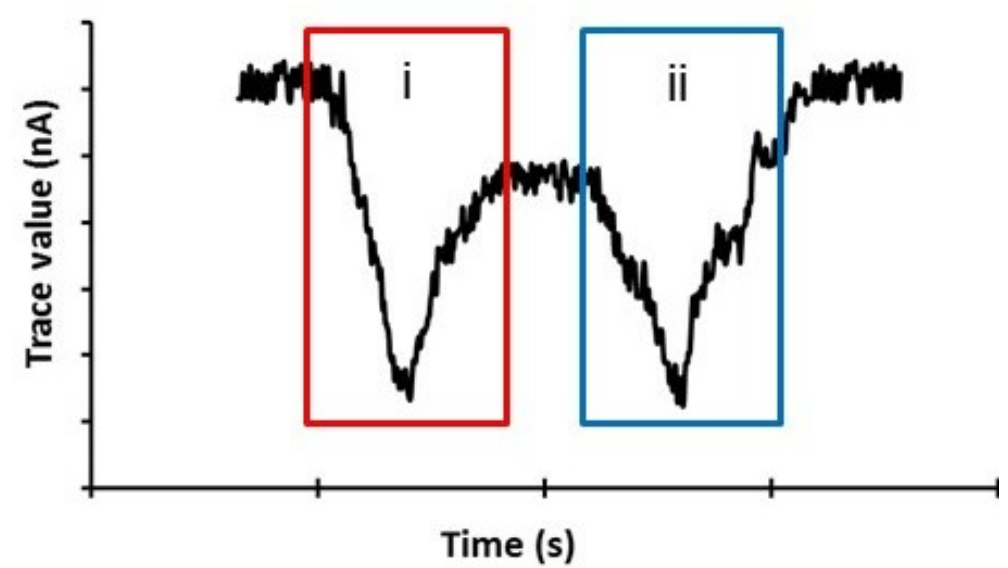

Figure 2: A scatter-plot displaying average rate of four experiments using $120 \mathrm{~nm}, 3 \times 10^{9}$ beads $/ \mathrm{mL}$, beads functionalised with VEa5 (anti-VEGF aptamer) against concentration of VEGF $_{165}$, circles represent raw data, diamonds are values where the double peaks have been removed as described within the methods section. The error bars show 1 Standard deviation from the mean. B Example peak trace for (i) a bead travelling in top-to-bottom (see supplementary figure 1 for more examples) direction and (ii) a bead travelling bottom-to-top, or one not completely traversing the pore i.e. Bottom-pore-bottom.

\section{VEGF and PDGF detection}

In the final assay the aim is to quantify both VEGF and PDGF simultaneously, we therefore chose to anchor each aptamer onto a different sized bead to allow the signals to be separated. Typically the VEGF aptamers were immobilised onto $120 \mathrm{~nm}$ beads and the PDGF aptamers onto 300nm beads, as shown in figure 1. 
Initially the TRPS instrument was set up with a positive bias applied to the electrode under the nanopore (lower fluid cell) similarly to that described in our previous work(Billinge et al., 2014). Samples containing $120 \mathrm{~nm}$ beads functionalised with VEa5 aptamer which had been incubated with several concentrations of VEGF 165 (as described in the experimental section) were introduced to the TRPS instrument; the resultant data is displayed in Figure $2 \mathrm{~A}$. Initially the rate was predicted to diminish with increasing protein concentration, as observed for thrombin protein during a similar experimental design (Billinge et al., 2014); this was thought to due to the protein binding to the DNA and shielding the charge on the phosphate backbone. As we increase the concentration of VEGF from 0 to $10 \mathrm{nM}$ we see the predicted decrease in particle rate, however at higher concentrations, $>10 \mathrm{nM}$, the particle rate increases before reaching a steady value of $\sim 220$ beads per minute. We examined the current traces in more detail and observed numerous double peaks, shown in figure 2Bii. The conical pore used in TRPS gives rise to a characteristic peak shape, displayed in figure 1 and explained in detail elsewhere(Willmott et al., 2012). The shape of the peak gives information on the direction which the particle is travelling. For beads travelling from the top of the fluid cell entering the narrow sensing zone first before then traveling through the pore into the expanding conical region they produce a peak shape as shown in figures 1 and 2Bi (more examples are displayed in supplementary figure 1). If a particle was to travel in the reverse direction the peak shape would be inverted (figure 2Bii and supplementary figure 1). Below $10 \mathrm{nM}$ no reverse peaks were observed throughout the experiment, however when the concentration of VEGF increased, > 10nm, there was an increase in the number of peak traces, termed "reverse peaks" which represent beads coming back up from the base of the pore (figure 2Bii). The presence of these reverse peaks led to an increase in the rate recorded by the software. When these erroneous peaks were then removed from the data set, the rate is seen to diminish, as expected, with increasing VEGF 165 concentration, see figure $2 \mathrm{~A}$ black diamonds. We repeated the experiment with a second aptamer, termed V7t1, and the same effect was observed (supplementary figure 2). The two selected anti-VEGF aptamers bind to different regions of the VEGF protein - VEa5 binds the heparin binding domain(Hasegawa et al., 2008) whereas V7t1 forms a g-quadruplex and interacts with the receptor binding domain of VEGF(Nonaka et al., 2010), both of these aptamers produced reversed peaks at higher analyte concentrations and provide evidence that this observation 
was not due to a specific aptamer-epitope interaction. We performed and rechecked an assay using the previously published thrombin aptamer (data not shown) and no reverse peaks were observed at higher thrombin concentrations.

In a typical TRPS experiment it has been shown that the number of bead translocations is a function of the bead concentration, diffusion coefficient of the beads, applied pressure, electroosmotic and electrophoretic mobility. Thus within the current experimental setup, ensuring concentration, pressure and osmotic contributions remain constant, changes in particle translocations can be inferred to a change in the electrophoretic mobility of the beads(Billinge et al., 2014). We attributed our observation of the beads re-entering the pore and moving from the lower fluid cell back up to the upper to the addition of the protein and its charge. VEGF $_{165}$ has a reported isoelectric point at pH 8.5(Ferrara et al., 1992), suggesting that at the $\mathrm{pH}$ of 7.4 the protein should have a net positive surface charge, whereas thrombin (pl 7.1(Righettl and Tudor, 1981)) would be largely neutral. Upon binding to the aptamer and shielding the phosphate backbone the charge upon the protein must therefore dominate the electrophoretic mobility. As this occurred with both VEGF aptamers, which as stated above bind to different regions of the protein, it does not seem to affect the results which epitope is occupied.

To test the hypothesis we switched the applied voltage bias to a negative value (negative electrode, in the lower fluid cell). In this configuration DNA modified beads usually do not traverse the pore opening, or have a much reduced frequency of translocation, here for the $120 \mathrm{~nm}$ particles no translocation events were recorded. Upon incubation with the VEGF protein the frequency of beads was directly proportional to the concentration of the protein, shown in Figure 3. This is a major improvement and observation from previous thrombin work(Billinge et al., 2014). In thrombin studies the number of translocation events per minute decreases as target analyte concentration increased, in effect switching off the signal. Here we have a more typical and convenient setup, wherein an increase in analyte concentration produced an increase in signal.

A series of experiments were then performed to confirm that PDGF could also be detected using this technique. First a positive bias was applied to the lower fluid cell of $+0.4 \mathrm{~V}$, and a diminution of particle rate was observed as PDGF concentration increases. Unlike within the VEGF experiment where beads were observed translocating the pore in reverse i.e. from the lower fluid cell to the upper, as 
displayed within supplementary figure 3 using $300 \mathrm{~nm}$ beads. To ascertain if this effect was dependent upon the bead size i.e. due to additional mass of the $300 \mathrm{~nm}$ beads creating bias given the larger gravity forces, the same experiment was performed using $120 \mathrm{~nm}$ beads. Again we failed to see any reverse translocations suggesting gravitational effects did not impact upon the measurement. The same trend was observed and is displayed in supplementary figure 4, confirming that the lack of reversed peaks is not an artefact of using a different bead size. As PDGF-BB has an isoelectric point of 9.8 it is assumed that the protein would have a positive surface charge in the chosen electrolyte. In order to confirm that the two bead populations could be analysed together under the same conditions, experiments were then performed under a fixed negative bias of $-0.4 \mathrm{~V}$. Supplementary figure 5 displays the results for these experiments in which a clear and proportional increase in rate is visible with increasing PDGF concentrations. As the behaviour of PDGF and VEGF bound beads was found to be similar, providing an increase in rate under a negative bias, it can be concluded that both proteins can be observed binding their aptamers under these conditions.

As each PDGF-BB molecule can bind two aptamers, aggregation is sometimes observed in their assays(Huang et al., 2005). Incontrast, addition of VEGF does not cause aggregation as the protein only has one epitope for the aptamer(Hasegawa et al., 2008). In our set of experiments we also observe an increase in the number of aggregates as PDGF-BB concentration increases up to $10 \mathrm{nM}$, as displayed in supplementary figures 6 and 7. However, an increase in rate in relation to PDGF-BB concentration is still observable, thus the formation of aggregates did not affect the AptaTRPS technique. 


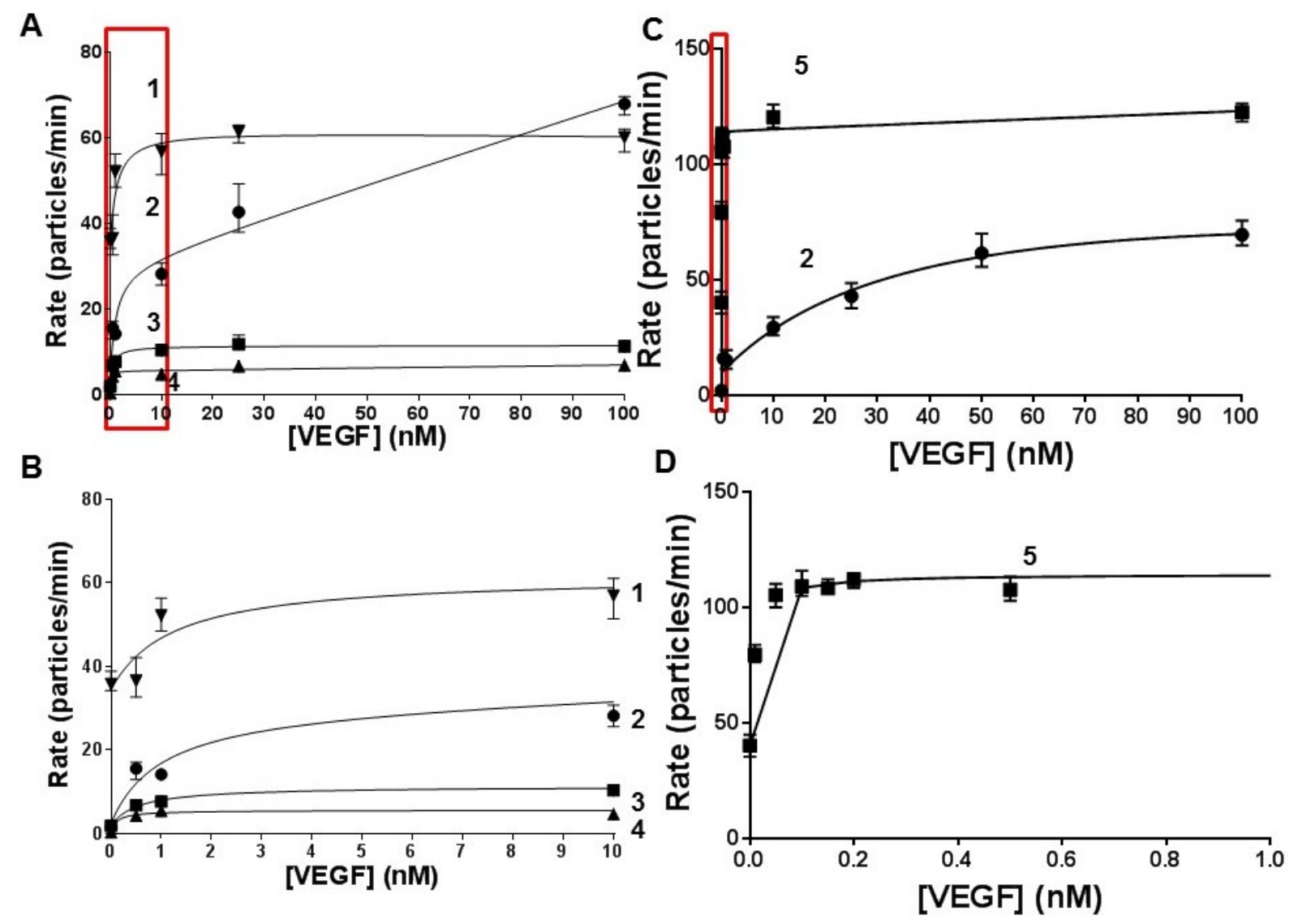

Figure 3: A Average rate for triplicate measurments against VEGF $\mathrm{F}_{165}$ concentration for (1) $3 \times$ $10^{9}$ beads $/ \mathrm{mL} 300 \mathrm{~nm}$ beads, (2) $3 \times 10^{9}$ beads $/ \mathrm{mL} 120 \mathrm{~nm}$ beads, (3) $2.5 \times 10^{9}$ beads $/ \mathrm{mL}$ $120 \mathrm{~nm}$ beads and (4) $2 \times 10^{9}$ beads/mL $120 \mathrm{~nm}$ beads all functionalised with VEa5 aptamer. B Expanded view of highlighted area in figure $3 \mathrm{~A}$. C Average rate for triplicate measurments against $V_{E G F} F_{165}$ concentration for $3 \times 10^{9}$ beads $/ \mathrm{mL} 120 \mathrm{~nm}$ beads functionalised with either (2) VEa5 or (5) V7t1 aptamer. D Expanded view of highlighted area in figure 3C. The error bars show 1 Standard deviation from the mean

\section{Factors which influence the sensitivity of the assay}

To determine if the bead size and concentration would have any impact on the sensitivity and ease of the assay we investigated their effects using the VEGF aptamers VEa5 and V7t1. Data summarising the effects of bead diameter and concentration are displayed in figure $3 \mathrm{~A}$ and $3 \mathrm{~B}$. To investigate the effect of bead concentration VEa5 modified beads at 3, 2.5 and $2 \times 10^{9}$ beads $/ \mathrm{mL}$ were added to solution containing the VEGF protein (figure 3A/B curves 2, 3 and 4). As the bead concentration decreases, so does the concentration of VEGF at which the bead rate remains constant $100 \mathrm{nM}$ (line 2) to $25 \mathrm{nM}$ (line 4), this has an impact upon the dynamic range of the assay and is likely due to a decrease in the number of available binding sites in solution, leading to the saturation of each individual bead 
with VEGF at much lower concentrations of protein. As indicated above the number of beads moving through the pore is proportional to their concentration, hence lower concentrations of beads will result in a lower overall rate, at bead concentrations below $2.5 \times 10^{9}$ beads $/ \mathrm{mL}$ it could be suggested that the bead count is so low $(<10$ beads a minute) it is no longer practical for accurate measurement and lengthens the required assay run-time unnecessarily.

To investigate the impact of bead size, the bead concentration was kept the same (3 $\times 10^{9}$ beads $/ \mathrm{mL}$ ) and the diameter was increased to $300 \mathrm{~nm}$ (figure $3 \mathrm{~A}$ and $\mathrm{B}$ line 1 and 2). The rate of $300 \mathrm{~nm}$ beads at $0 \mathrm{nM}$ VEGF was initially higher than the $120 \mathrm{~nm}$ beads with average rates of 35 and 0 beads/min respectively. It is likely that this is due to both the increased mass of the beads, leading to a higher gravitational effect, and also due to a lower relative loading of DNA per area of beads suspended. Both beads have comparable binding capacities (see method section) therefore we conclude the $300 \mathrm{~nm}$ beads have a lower packing density of streptavidin, and therefore DNA, on their surface.

The differences in packing density might also be responsible for the differences in rate signal between 120 and $300 \mathrm{~nm}$ beads. It is observed that there is a sharp initial increase in rate upon addition of VEGF and the rate signal appears to saturate sooner for the $300 \mathrm{~nm}$ beads; this could indicate that the sensitivity could be tailored by altering the packing density of capture probe on the surface of beads. For the purposes of this assay, these results demonstrate that the $300 \mathrm{~nm}$ beads are able to provide a rate-related signal change in response to protein binding which can be analysed via TRPS, further supporting the notion of the multiplexed AptaTRPS assay. Figure $3 C$ represents the difference in the rate signal for two anti-VEGF aptamers: VEa5 (line 2) and V7t1 (line 5). There are two observations from this data; firstly, the rate signal for the V7t1 aptamer increases at low concentrations of VEGF and appears to have levelled out by $10 \mathrm{nM}$, whereas the rate of VEa5 beads continues to increase up to $100 \mathrm{nM}$ of added protein. Initially we explored the same VEGF concentrations for both aptamers, however due to the rate remaining constant for the V7t1 aptamer at concentrations of VEGF $>1 \mathrm{nM}$ we chose to significantly reduce the amount of VEGF added. To illustrate this effect, Figure 3D displays an expanded view of the data for the V7t1 aptamer which exemplifies the ability of this aptasensor to detect VEGF at concentrations down to $0.05 \mathrm{nM}$. This is due to differences in aptamer binding mechanism and affinity. VEa5 has a reported $K_{D}$ of 130 
$\mathrm{nM}($ Hasegawa et al., 2008) and is postulated to bind due to the presence of stemloop structures(Kanakaraj et al., 2013). V7t1 has a reported $K_{D}$ of $1.4 \mathrm{nM}$ for VEGF $_{165}$ and due to its lack of selectivity over VEGF 121 is postulated to bind the receptor binding domain present on both VEGF analogues by forming a Gquadruplex(Nonaka et al., 2010); the superior affinity of V7t1 allows for the measurement of smaller concentrations of VEGF 165 on this platform.

The second observation from figure $3 \mathrm{C}$ is the marked difference in maximum rate between VEa5 and V7t1 which reach circa 60 beads/minute and 120 beads/minute, respectively at higher VEGF concentrations. As the overall number of aptamer molecules and beads should be the same, the difference in rate must be due to the difference in length of DNA. As demonstrated in our previous work, additional to the concentration of DNA added to the beads surface, the length of DNA sequence used is also a key factor in determining the maximum bead rate through the pore(Billinge et al., 2014). The V7t1 aptamer is 20 nucleotides shorter than VEa5 and with a negative bias under the pore, and the concentration of the beads being identical in all experiments the rates vary $\sim 40$ beads/minute (V7t1) and $\sim 1$ bead/minute (VEa5) respectively, when VEGF concentration is $0 \mathrm{nM}$. This is attributed to the effects of the length of the DNA, with the short DNA sequence able to pass through the pore more easily. Upon the protein binding to the aptamer, we believe there are two possible reasons for the higher total rate for the shorter aptamer sequence. The first could be due to the protein shielding the phosphate backbone to a greater extent for the shorter DNA, and secondly the binding mechanism. The conformation of the DNA aptamer, the location it holds the protein with respect to the bead, could impact on the bead zeta potential and drag, however further work is needed to infer implications of any mechanistic differences.

To further support the theory that bead rate is dominated by the charge of VEGF, we then altered the $\mathrm{pH}$ of our buffer to 9.3. It was expected that at this $\mathrm{pH}$ the VEGF would have an overall negative surface charge, and with a negative voltage bias applied under the pore the previously observed increase in particle rate with increasing protein concentration would be eliminated. As shown in table 1 this was indeed found to be the case, with bead rate remaining at 0.5 beads $/ \mathrm{min}$. When the applied potential was revered to an equal positive potential, the rate decreases with increasing protein concentration, reverting back to the trend 
observed previously with thrombin(Billinge et al., 2014), i.e. a diminution, suggesting a charge-shielding mechanism is taking place.

\begin{tabular}{|l|l|l|l|l|}
\cline { 2 - 5 } \multicolumn{1}{c|}{} & \multicolumn{3}{c|}{ Rate (beads/min) } & \multicolumn{2}{c|}{ Positive bias (+ 0.4 V) } \\
\cline { 2 - 5 } \multicolumn{1}{c|}{} & Negative bias (- 0.4 V) & $\mathrm{pH} \mathrm{7.4}$ & $\mathrm{pH} \mathrm{9.3}$ \\
\hline [VEGF] (nM) & $\mathrm{pH} \mathrm{7.4}$ & $\mathrm{pH} \mathrm{9.3}$ & 150 & 165 \\
\hline 0 & 1.6 & 0.5 & 40.8 & 90 \\
\hline 100 & 68 & 0.5 & 0.8 & \\
\hline
\end{tabular}

Table 1: comparative summary of the effect of buffer $\mathrm{pH}$ on recorded particle rate using the same pore and instrument conditions. 


\section{Filtering the data sets from two different particles}

In order to successfully multiplex the rate signals for two different bead sizes, it first must be possible to differentiate the beads from one another. Figure 4A displays the calibrated diameter distributions for the two different bead sets measured separately and together (inset) which confirms that the resolution of TRPS is able to distinguish these two bead populations without overlap. The distribution of $120 \mathrm{~nm}$ bioadembeads streptavidin plus (Figure 4Ai) is much narrower than for the $300 \mathrm{~nm}$ bioadembeads streptavidin; this is believed to be due to a difference in the stringency of bead manufacture - it is possible to see the distribution of bead diameters with great accuracy due to the particle-by-particle analysis of TRPS which has been shown to have far greater resolving power over conventional sizing techniques, such as Dynamic Light Scattering, when analysing non-uniform dispersions(Henriquez et al., 2004; Roberts et al., 2012; Willmott et al., 2010). 

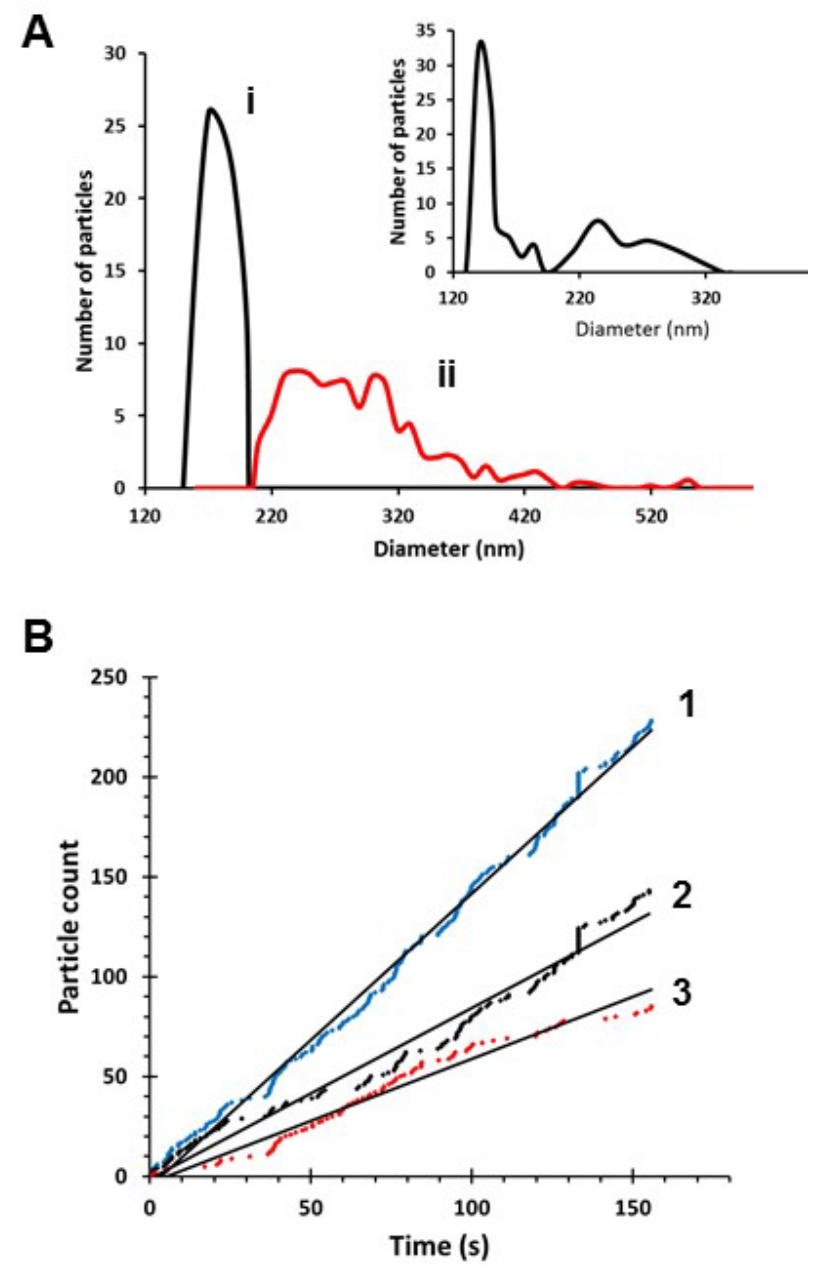

Figure 4: A Distribution of particle diameters for (i) $120 \mathrm{~nm}$ bio-adembeads streptavidin plus (Ademtech, France) and (ii) $300 \mathrm{~nm}$ bio-adembeads streptavidin (Ademtech, France). B 1 Combined rate of equal concentrations of 120 and $300 \mathrm{~nm}$ beads passing through the pore, 2 rate of $120 \mathrm{~nm}$ beads taken from 1,3 rate of $300 \mathrm{~nm}$ beads, taken from 1 .

\section{Multiplexing PDGF-BB and VEGF measurement}

Figure $4 \mathrm{~B}$ line 1 is an example of the raw data plot showing particle count versus time for a sample containing both 120 and $300 \mathrm{~nm}$ beads. Following the experiment we can reanalyse the data and divide the total bead count into the two different beads sizes, lines 2 and 3 . The $120 \mathrm{~nm}$ beads (line 2) are seen to run through the pore at a greater frequency than the $300 \mathrm{~nm}$ beads, despite being at the same concentration; this has been attributed to the effect of the ratio of the diameter of the pore constriction vs the diameter of the beads. As the pore size is reduced the particle rate decreases(Kozak et al., 2012) and this will be seen to a greater extent for the larger $300 \mathrm{~nm}$ beads. We were unable to increase the size of the pore any 
further as the signal from the smaller beads would have been lost in the baseline noise.

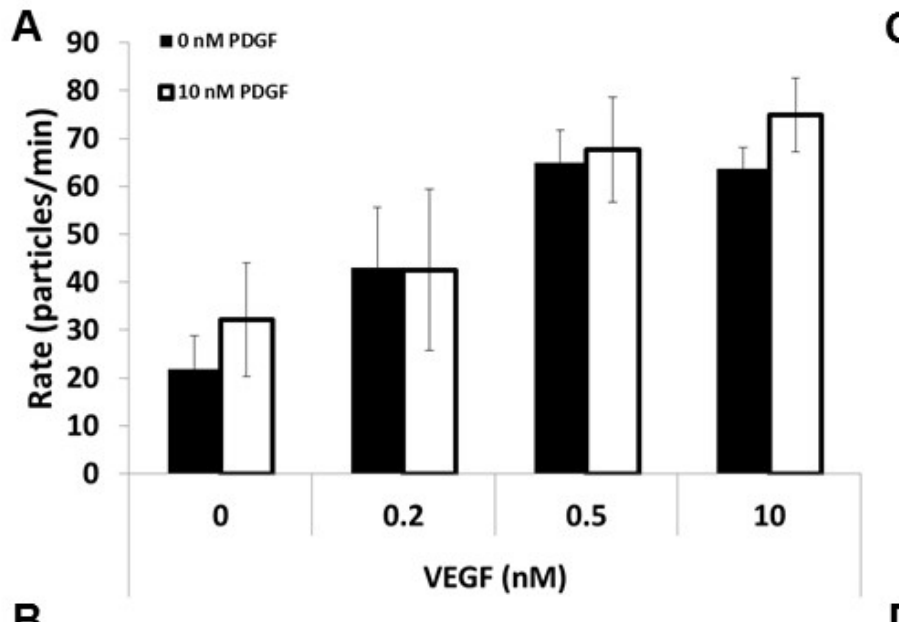

C

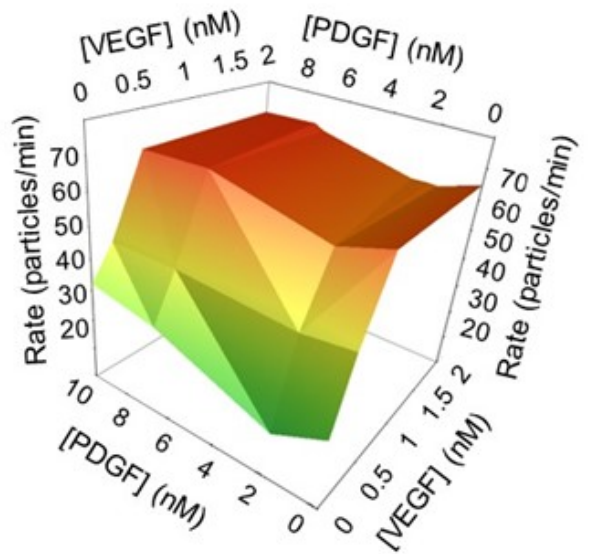

B

D
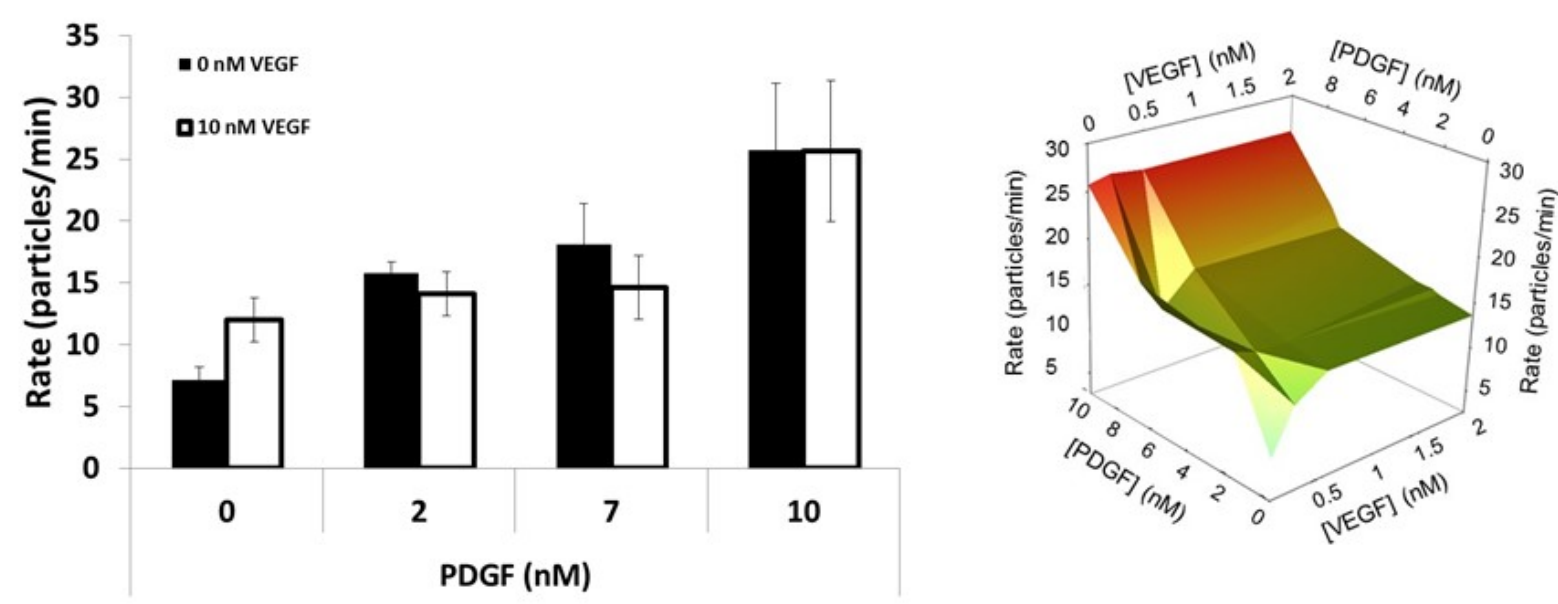

Figure 5: A bar graph of triplicate experiments comparing the average particle rates of $120 \mathrm{~nm}$ beads functionalised with V7t1 as VEGF ${ }_{165}$ concentration increases with and without the addition of $10 \mathrm{nM}$ PDGF-BB in the presence of $300 \mathrm{~nm}$ beads functionalised with anti-PDGF aptamer. B bar graph of triplicate experiments comparing the average particle rates of $300 \mathrm{~nm}$ beads functionalised with anti-PDGF aptamer as PDGF-BB concentration increases with and without the addition of $10 \mathrm{nM} \mathrm{VEGF}{ }_{165}$ in the presence of $120 \mathrm{~nm}$ beads functionalised with V7t1. C 3D surface plot displaying the effect of PDGF and VEGF on particle rate of $120 \mathrm{~nm}$ beads. D 3D surface plot displaying the effect of PDGF and VEGF on particle rate of $300 \mathrm{~nm}$ beads. Error bars represent 1 standard deviation from the mean.

In order to demonstrate its ability to multiplex, a control experiment was devised to ensure that the presence of additional proteins and beads do not interfere with the signal. Figure $5 \mathrm{~A}$ shows the rate of $120 \mathrm{~nm}$ beads in the presence of different concentrations of VEGF with and without the secondary analyte - 10 nM PDGF-BB. Figure 5B displays the rate for $300 \mathrm{~nm}$ beads in the presence of different 
concentrations of PEGD with and without the secondary analyte - $10 \mathrm{nM} \mathrm{VEGF} 165$. These experiments illustrate that the signal from one particle does not change or deviate in the presence of additional beads and target proteins. There does seem to be a small effect of the VEGF protein on the PDGF assay, figure 5b, where at 0nM PDGF and 10nM VEGF there is an increase in particle rate, and a decrease in sensitivity to PDGF at lower concentrations recorded as a small change in particle rate for PDGF concentrations 2-7 nM. This might be attributed to nonspecific interactions between the PDGF aptamer and the VEGF target, and that these nonspecific or weaker interactions are overcome at higher concentrations of PDGF.

This is further illustrated in supplementary figure 8 which displays the rate of $120 \mathrm{~nm}$ beads functionalised with Vt71 aptamer against concentration of VEGF 165 in the presence of an equal concentration of $300 \mathrm{~nm}$ anti-PDGF aptamer loaded beads with several concentrations of PDGF-BB ranging from $0-10 \mathrm{nM}$. An increase in rate is still visible for the $120 \mathrm{~nm}$ beads regardless of the addition of $300 \mathrm{~nm}$ beads and PDGF-BB concentrations. As illustrated in figure 3, figure $5 \mathrm{~A}$ verifies that no significant difference between the values for 0.5 and $10 \mathrm{nM}$ of VEGF, suggesting that at this point all of the available binding sites on the $120 \mathrm{~nm}$ bead are occupied. The frequency of both bead sets was measured in the presence of several combinations of concentrations of PDGF-BB and VEGF 165 . The data from all of these experiments are represented as surface plots in Figure 5C and D. For both bead sets (C and D) rate is seen to increase when their specific protein target concentration is increased. This trends remains largely unaffected by the presence of the other bead population and protein and the two can be measured simultaneously.

These results suggest that the simultaneous measurement of two different growth factors by TRPS is possible by using differently sized beads to provide a differentiable signal which is able to be selectively filtered by blockade magnitude to pull out the desired individual rate data. The observed increases in particle rate are believed to be due to an increase in electrophoretic mobility toward the negative electrode at the base of the pore when the positive proteins bind selectively to their respective DNA aptamers via a conformational change.

Multiplexing the TRPS method illustrates the potential for the platform to be used as a biosensor. As demonstrated in our previous work(Billinge et al., 2014) the 
technique offers comparable sensitivity and assay times when compared to Surface Plasmon Resonance techniques or flow cytometry platforms. The TRPS technique does not have the throughout put of a modern flow cytometer but does offer an improved signal and resolution sub 500nm particles, without the need of fluorescent labels.

\section{Conclusions}

VEGF $_{165}$ and PDGF-BB were successfully monitored binding to aptamer-laden beads of 120 and $300 \mathrm{~nm}$ in diameter. The signals generated were able to be monitored in situ and also in tandem with both proteins measured independently from one another using the same sample using the beads themselves as a label, without the need for any additional identifiers such as fluorophores. Parameters regarding bead concentration and size were considered and a multiplexed assay undertaken.

\section{Acknowledgments}

The authors thank the Centre for Analytical Science at Loughborough University. The work was supported by the European Commission for Research (PCIG11-GA-2012-321836 Nano4Bio) and Loughborough University Chemistry Department (Start-up fund). The research was supported by the National Institute for Health Research (NIHR) Diet, Lifestyle \& Physical Activity Biomedical Research Unit based at University Hospitals of Leicester and Loughborough University. The views expressed are those of the authors and not necessarily those of the NHS, the NIHR or the Department of Health. 


\section{References}

Belgore, F.M., Lip, G.Y., Bareford, D., Wadley, M., Stonelake, P., Blann, a D., 2001. Plasma levels of vascular endothelial growth factor (VEGF) and its receptor, Flt1, in haematological cancers: a comparison with breast cancer. Am. J. Hematol. 66, 59-61. doi:10.1002/1096-8652(200101)66:1<59::AID-AJH1011>3.0.CO;2-Z

Billinge, E.R., Broom, M., Platt, M., 2014. Monitoring aptamer-protein interactions using tunable resistive pulse sensing. Anal. Chem. 86, 1030-7. doi:10.1021/ac401764c

Chan, C., Mak, W., Cheung, K., Sin, K., Yu, C., Rainer, T., Renneberg, R., 2013. Evidence-based PointOof-Care Diagnostics: Current Status and Emerging Technologies. Annu. Rev. Anal. Chem. 6, $191-211$.

Chiu, T.-C., Huang, C.-C., 2009. Aptamer-functionalized nano-biosensors., Sensors (Basel, Switzerland). doi:10.3390/s91210356

Cho, E.J., Lee, J.-W., Ellington, A.D., 2009. Applications of aptamers as sensors. Annu. Rev. Anal. Chem. (Palo Alto. Calif). 2, 241-64. doi:10.1146/annurev.anchem.1.031207.112851

de-los-Santos-Álvarez, N., Lobo-Castañón, M.J., Miranda-Ordieres, A.J., TuñónBlanco, P., 2008. Aptamers as recognition elements for label-free analytical devices. TrAC Trends Anal. Chem. 27, 437-446. doi:10.1016/j.trac.2008.03.003

Deng, K., Xiang, Y., Zhang, L., Chen, Q., Fu, W., 2013. An aptamer-based biosensing platform for highly sensitive detection of platelet-derived growth factor via enzyme-mediated direct electrochemistry. Anal. Chim. Acta 759, 61 65.

Famulok, M., Mayer, G., 2011. Aptamer modules as sensors and detectors. Acc. Chem. Res. 44, 1349-58. doi:10.1021/ar2000293

Fang, X., Tan, W., 2010. Aptamers genereated from cell-SELEX for molecular medicine: A chemical biology approach. Acc. Chem. Res. 43, 48-57. doi:10.1021/ar900101s.Aptamers

Ferrara, N., Houck, K., Jakeman, L., Leung, D.W., 1992. Molecular and biological properties of the vascular endothelial growth factor family of proteins. Endocr. Rev. 13, $18-32$.

Hamilton, T.D., Leugner, D., Kopciuk, K., Dixon, E., Sutherland, F.R., Bathe, O.F., 2014. Identification of prognostic inflammatory factors in colorectal liver metastases. BMC Cancer 14, 542. doi:10.1186/1471-2407-14-542

Hasegawa, H., Sode, K., Ikebukuro, K., 2008. Selection of DNA aptamers against VEGF165 using a protein competitor and the aptamer blotting method.

Biotechnol. Lett. 30, $829-834$. 
Henriquez, R.R., Ito, T., Sun, L., Crooks, R.M., 2004. The resurgance of coulter counting for analysing nanoscale objects. Analyst 129, 478-482.

Huang, C.-C., Huang, Y.-F., Cao, Z., Tan, W., Chang, H.-T., 2005. Aptamer-modified gold nanoparticles for colorimetric determination of platelet-derived growth factors and their receptors. Anal. Chem. 77, 5735-41. doi:10.1021/ac050957q

Jelkmann, W., 2001. Pitfalls in the measurement of circulating vascular endothelial growth factor. Clin. Chem. 47, 617-23.

Kanakaraj, I., Chen, W.-H., Poongavanam, M., Dhamane, S., Stagg, L.J., Ladbury, J.E., Kourentzi, K., Strych, U., Willson, R.C., 2013. Biophysical characterization of VEGF-aHt DNA aptamer interactions. Int. J. Biol. Macromol. 57, 69-75. doi:10.1016/j.ijbiomac.2013.02.016

Karayiannakis, A.J., N, S.K., Polychronidis, A., Zbar, A., Kouraklis, G., Simopoulos, C., Karatzas, G., 2002. Circulating VEGF Levels in the Serum of Gastric Cancer Patients. Ann. Surg. 236, 37-42. doi:10.1097/01.SLA.0000018567.54479.E6

Kozak, D., Anderson, W., Trau, M., 2012. Tuning Particle Velocity and Measurement Sensitivity by Changing Pore Sensor Dimensions. Chem. Lett. 41, 1134-1136. doi:10.1246/cl.2012.1134

Ledur, A., Fitting, C., David, B., Hamberger, C., Cavaillon, J., 1995. Variable estimates of cytokine levels produced by commercial ELBA kits : results using international cytokine standards 186, 171-179.

Levy-Nissenbaum, E., Radovic-Moreno, A.F., Wang, A.Z., Langer, R., Farokhzad, O.C., 2008. Nanotechnology and aptamers: applications in drug delivery. Trends Biotechnol. 26, 442-9. doi:10.1016/j.tibtech.2008.04.006

Li, L., Wang, L., Zhang, W., Tang, B., Zhang, J., Song, H., Yao, D., Tang, Y., Chen, X., Yang, Z., Wang, G., Li, X., Zhao, J., Ding, H., Reed, E., Li, Q.Q., 2004. Correlation of serum VEGF levels with clinical stage, therapy efficacy, tumor metastasis and patient survival in ovarian cancer. Anticancer Res. 24, 1973-9.

Madsen, C.V., Steffensen, K.D., Olsen, D.A., Waldstrøm, M., Smerdel, M., Adimi, P., Brandslund, I., Jakobsen, A., 2012. Serial measurements of serum PDGF-AA, PDGF-BB, FGF2, and VEGF in multiresistant ovarian cancer patients treated with bevacizumab. J. Ovarian Res. 5, 23. doi:10.1186/1757-2215-5-23

Matsumoto, T., Mugishima, H., 2006. Signal transduction via vascular endothelial growth factor (VEGF) receptors and their roles in atherogenesis. J. Atheroscler. Thromb. 13, 130-5.

Mayer, G., 2009. The chemical biology of aptamers. Angew. Chem. Int. Ed. Engl. 48, 2672-89. doi:10.1002/anie.200804643

Nonaka, Y., Sode, K., Ikebukuro, K., 2010. Screening and improvement of an antiVEGF DNA aptamer. Molecules 15, 215-25. doi:10.3390/molecules15010215 
Platt, M., Willmott, G.R., Lee, G.U., 2012. Resistive pulse sensing of analyte-induced multicomponent rod aggregation using tunable pores. Small 8, 2436-44. doi:10.1002/smll.201200058

Righettl, P.G., Tudor, G., 1981. Isoelectric points and molecular weights of proteins: a new table. J. Chromatogr. 220, 115-194.

Roberts, G.S., Kozak, D., Anderson, W., Broom, M., Vogel, R., Trau, M., 2010. Tunable nano/micropores for particle detection and discrimination: scanning ion occlusion spectroscopy. Small 6, $2653-2658$.

Roberts, G.S., Yu, S., Zeng, Q., Chan, L.C.L., Anderson, W., Colby, A.H., Grinstaff, M.W., Reid, S., Vogel, R., 2012. Tunable pores for measuring concentrations of synthetic and biological nanoparticle dispersions. Biosens. Bioelectron. 31, 1725. doi:10.1016/j.bios.2011.09.040

Rotem, D., Jayasinghe, L., Salichou, M., Bayley, H., 2012. Protein detection by nanopores equipped with aptamers. J. Am. Chem. Soc. 134, 2781-7. doi:10.1021/ja2105653

Rowe, W., Platt, M., Day, P.J.R., 2009. Advances and perspectives in aptamer arrays. Integr. Biol. (Camb). 1, 53-8. doi:10.1039/b815539a

Song, Y., Huang, Y.-Y., Liu, X., Zhang, X., Ferrari, M., Qin, L., 2014. Point-of-care technologies for molecular diagnostics using a drop of blood. Trends Biotechnol. 32, 132-9. doi:10.1016/j.tibtech.2014.01.003

Tarkowski, E., Issa, R., Sjo, M., Wallin, A., Blennow, K., 2002. Increased intrathecal levels of the angiogenic factors VEGF and TGF- $\wedge_{\llcorner}$in Alzheimer' $s$ disease and vascular dementia 23, 237-243.

Tombelli, S., Minunni, M., Mascini, M., 2005. Analytical applications of aptamers. Biosens. Bioelectron. 20, 2424-34. doi:10.1016/j.bios.2004.11.006

Vogel, R., Anderson, W., Eldridge, J., Glossop, B., Willmott, G., 2012. A variable pressure method for characterizing nanoparticle surface charge using pore sensors. Anal. Chem. 84, 3125-31. doi:10.1021/ac2030915

Vogel, R., Willmott, G., Kozak, D., Roberts, G.S., Anderson, W., Groenewegen, L., Glossop, B., Barnett, A., Turner, A., Trau, M., 2011. Quantitative sizing of nano/microparticles with a tunable elastomeric pore sensor. Anal. Chem. 83, 3499-506. doi:10.1021/ac200195n

Wei, L.-H., Kuo, M.-L., Chen, C.-A., Chou, C.-H., Lai, K.-B., Lee, C.-N., Hsieh, C.-Y., 2003. Interleukin-6 promotes cervical tumor growth by VEGF-dependent angiogenesis via a STAT3 pathway. Oncogene 22, 1517-27. doi:10.1038/sj.onc.1206226 
Willmott, G.R., Platt, M., Lee, G.U., 2012. Resistive pulse sensing of magnetic beads and supraparticle structures using tunable pores. Biomicrofluidics 6, 141031410315. doi:10.1063/1.3673596

Willmott, G.R., Vogel, R., Yu, S.S.C., Groenewegen, L.G., Roberts, G.S., Kozak, D., Anderson, W., Trau, M., 2010. Use of tunable nanopore blockade rates to investigate colloidal dispersions. J. Phys. Condens. Matter 22, 454116. doi:10.1088/0953-8984/22/45/454116

Yager, P., Domingo, G.J., Gerdes, J., 2008. Point-of-care diagnostics for global health. Annu. Rev. Biomed. Eng. 10, 107-44.

doi:10.1146/annurev.bioeng.10.061807.160524 